\title{
Atmospheric parameters of a sample of giant stars with accurate radial velocity variations
}

\author{
L. da Silva ${ }^{1}$, L. Girardi ${ }^{2}$, L. Pasquini ${ }^{3}$, M. Döllinger ${ }^{3}$, R. De

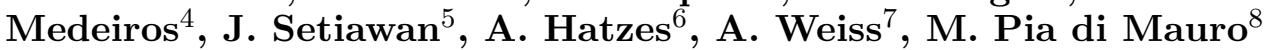 \\ ${ }^{1}$ Observatório Nacional, Rio de Janeiro, Brazil, email: licio@on.br; ${ }^{2}$ INAF-Oss.Ast di Trieste, \\ Italy; ${ }^{3}$ ESO Garching, Germany; ${ }^{4}$ UFRN, Natal, Brazil, ${ }^{5}$ MPI Heidelberg, Germany; \\ ${ }^{6}$ Thüringer Landessternwarte Tautemburg, Germany; ${ }^{7}$ MPA Garching, Germany; \\ ${ }^{8}$ INAF-Oss.Ast di Catania, Italy
}

Keywords. Stars: atmospheres, stars: fundamental parameters, stars: evolution

\section{Introduction}

In da Silva et al. (2005) is presented the detailed spectroscopic analysis of 60 evolved stars, which were previously studied for accurate radial velocity variations (Setiawan et al. 2003). The observations were made with FEROS and the $1.52 \mathrm{~m}$ ESO telescope of La Silla, partially in the ESO-Observatório Nacional (Brazil) agreement. The main goal of this study is to determine precise atmospheric fundamental data (Teff, log g, $[\mathrm{Fe} / \mathrm{H}]$ and micro-turbulence velocity) to derive ages and masses of the stars, trying to link them to the detected radial velocity variabilities. Here, due the short space at our disposal, we discuss only the effective temperature determination, comparing the values obtained from the excitation equilibrium of $\mathrm{FeI}$ and from $(\mathrm{V}-\mathrm{K})$ index with those from $(\mathrm{B}-\mathrm{V})$.

\section{Method}

The spectroscopic analysis was made in LTE, using the well known code of Monique Spite (Spite 1967). Some programs were developed to pass data to the code and to analyse its results, making the set automatic and fast, proper to study a large sample of stars. The stellar fundamental parameters were determined from the spectroscopic data in the usual way, that is: to determine the effective temperature Teff, we impose that the Fe abundance does not depend on the excitation potential; the microturbulence velocity is determined imposing that the $\mathrm{Fe}$ abundance is independent of the equivalent widths. The FeI/FeII ionization equilibrium has been used to determine the gravity. The method used is described in detail by del Peloso, da Sila, Porto de Mello (2005), with the difference that in this work the temperature is also a free parameter. MARCS Edwardsson et al. 1993 plane parallel atmosphere models are used. Equivalent widths were measured by using the DAOSPEC package (Pancino and Stetson 2005, in preparation), being the result checked and confirmed by measuring manually some lines of some stars.

\section{Discussion and Results}

When analyzing sub-giant and dwarf stars, it is preferred to determine Teff using colors indices and $\mathrm{H} \alpha$ and $\mathrm{H} \beta$ profiles (see, for ex., del Peloso, da Sila, Porto de Mello (2005)). But for the G-K giant stars the Balmer lines profiles are not useful and the best photometric index is $(\mathrm{V}-\mathrm{K})$. The only source of $\mathrm{K}$ (and $\mathrm{J}$ and $\mathrm{K}$ ) colors for all stars of our sample is the 2MASS catalog (Cutri et al. 2003). It is known that (B-V) is not a good Teff index for cool giants, but is precise enough to give the Teff initial value, necessary to start our spectroscopic process to determine this atmospheric parameter. The stars Teff from $(\mathrm{B}-\mathrm{V})$ and $(\mathrm{V}-\mathrm{K})$ indices were 

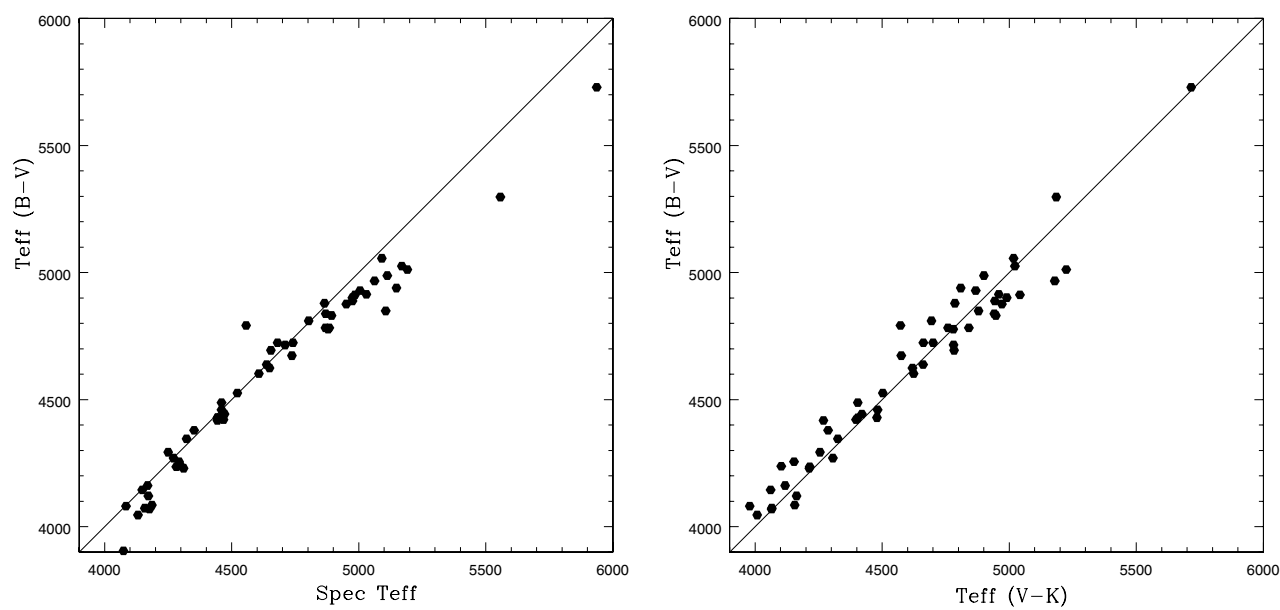

Figure 1. Teff from (B-V) compared with Teff from the spectroscopic analyses, on the left, and with Teff from $(\mathrm{V}-\mathrm{K})$.

determined using the Alonso et al. (1999) calibrations, for the stars with $\log g<3.5$, and the Alonso et al. (1996) calibration for the others. The 2MASS K color was converted to the CTS system (Alonso et al. (1998)) passing by the CIT system (Cutri et al. 2003). The (V-K) indices of the stars with $\log \mathrm{g}>3.5$ were converted to the Johnson system using the relation given by Alonso et al. (1998).

In Figure 1a) spectroscopic vs. photometric temperatures derived from B-V are plotted. In spite of some systematic trend, the scatter around the relationship is very small. In Figure 1b) we compare the B-V and V-K Teff; the scatter here is much larger and most of it can be attributed to the (V-K) Teff. This indicates that the 2MASS colors are not adequate for accurate determination of Teff for our sample stars. This is likely because our targets are very bright and they are above, or close to the saturation limit of the survey.

\section{Acknowledgements}

L da Silva acknowledges François and Monique Spite for having introduced him in the stellar spectroscopic research and for a friendship of many years.

\section{References}

Alonso, A., Arribas, R., \& Martinez-Roger, C. 1996, A\&SA 313, 873

Alonso, A., Arribas, R., \& Martinez-Roger, C. 1998, A\&A Supl Ser 131, 209

Alonso, A., Arribas, R., \& Martinez-Roger, C. 1999, A\&\&A Suppl. Ser 140, 261

Cutri, R. et al. , http://www.ipac.caltech.edu/2mass/releases/allsky/doc/sec6_4b.html 2003 (last update)

da Silva L., Girardi, L., Pasquini, L., Setiawan, J., Hatzes, A.P., De Medeiros, J.R., Dllinger, M., Weiss, A., \& Pia di Mauro, M. 2005, $A \& A$ submitted

del Peloso, E.F., da Silva, L., \& Porto de Mello, G.F. 2005, A\&JA 434, 275

Edvardsson, B., Andersen, J., Gustaffson, B. et al. 1993, A\&\&A 275, 101

Setiawan, J., Pasquini, L., da Silva, L., von der Lhe, O., \& Hatzes, A. 2003, A\&\&A 397, 1151

Spite, M. 1967, AnAp 30, n. 2, 1 